\title{
ALPPS for hepatocarcinoma under cirrhosis: a feasible alternative to portal vein embolization
}

\author{
Victor Lopez-Lopez ${ }^{1}$, Ricardo Robles-Campos ${ }^{1}$, Roberto Brusadin ${ }^{1}$, Asunción Lopez-Conesa ${ }^{1}$, \\ Jesus de la Peña ${ }^{2}$, Albert Caballero ${ }^{2}$, Julio Arevalo-Perez ${ }^{3}$, Alvaro Navarro-Barrios ${ }^{1}$, Paula Gómez ${ }^{1}$, \\ Pascual Parrilla-Paricio ${ }^{1}$
}

${ }^{1}$ Department of General, Visceral and Transplantation Surgery, ${ }^{2}$ Department of Pathology, Clinic and University Hospital Virgen de la Arrixaca, IMIB-ARRIXACA, Murcia, Spain; ${ }^{3}$ Radiology Department, Memorial Sloan Kettering Cancer Center, New York, NY, USA

Contributions: (I) Conception and design: V Lopez-Lopez, R Robles-Campos, PP Paricio; (II) Administrative support: R Brusadin, A Lopez-Conesa, J de la Peña; (III) Provision of study materials or patients: A Caballero, J Arevalo-Perez, A Navarro-Barrios, P Gomez; (IV) Collection and assembly of data: V Lopez-Lopez, R Robles-Campos, R Brusadin, A Lopez-Conesa, J de la Peña, A Caballero, J Arevalo-Perez, A Navarro-Barrios, P Gomez; (V) Data analysis and interpretation: V Lopez-Lopez, R Robles-Campos; (VI) Manuscript writing: All authors; (VII) Final approval of manuscript: All authors. Correspondence to: Ricardo Robles-Campos, MD, PhD. Department of General, Visceral and Transplantation Surgery, Clinic and University Hospital Virgen de la Arrixaca, Ctra. Madrid-Cartagena, s/n, 30120, El Palmar, Murcia, Spain. Email: rirocam@um.es.

\begin{abstract}
Hepatocellular carcinoma (HCC) is one of the most common and malignant tumors. Preoperative portal vein embolization (PVE) is currently the most accepted treatment before major hepatic resection for HCC in patients with liver fibrosis or cirrhosis and associated insufficient future liver remnant (FLR). In the last decade, associating liver partition and portal vein ligation for staged hepatectomy (ALPPS) technique has been described to obtain an increase of volume regarding PVE and a decrease of drop out. The initial excessive morbidity and mortality of this technique have decreased drastically due to a better selection of patients, the learning curve and the use of less aggressive variations of the original technique in the first stage. For both techniques a complete preoperative assessment of the FLR is the most important issue and only patients with and adequate FLR should be resected. ALPPS could be a feasible technique in very selected patients with HCC and cirrhosis. As long as it is performed in an experienced center could be used as a first choice technique versus PVE or could be used as a rescue technique in case of PVE failure.
\end{abstract}

Keywords: ALPPS; portal vein embolization (PVE); tourniquet-ALPPS; hepatocellular carcinoma (HCC); cirrhosis

Submitted Aug 05, 2019. Accepted for publication Aug 22, 2019.

doi: $10.21037 /$ atm.2019.10.57

View this article at: http://dx.doi.org/10.21037/atm.2019.10.57

\section{Introduction}

Hepatocellular carcinoma (HCC) is one of the most common and malignant tumors with incidence rate ranking the fifth and mortality rate the third among the malignant tumors in the world $(1,2)$. In cases, due to liver cirrhosis, sequential progression from fibrosis to cirrhosis culminates in HCC due to the preneoplastic setting of the cirrhotic background provides a conductive environment for cellular transformation $(3,4)$.
At present, the treatments for HCC include mainly liver resection, liver transplantation, radiofrequency ablation, transcatheter arterial chemoembolization (TACE), and drug therapy. In case of liver cirrhosis, liver transplantation is the most accepted surgical treatment of HCC, especially in Child B or C stages because it treats both, liver insufficiency and the tumor $(5,6)$. Shortage of grafts is the reason why liver resection is accepted as the first-line of treatment in patients with very early stage (7). Since most patients with HCC have underlying chronic liver disease or cirrhosis, the 
question of insufficient future liver remnant (FLR) is critical in this cohort to prevent postoperative liver failure (PHLF) $(8,9)$. Most of the cirrhotic patients have an intermediate or advanced stage of the Barcelona Clinic Liver Classification (BCLC), and in cases with well-preserved liver function the only indicated treatments are TACE or Sorafenib (10). Although the results in the literature are controversial, there are some studies with great outcomes in liver cirrhosis and advanced HCC and recently, some authors changed these criteria and claimed that liver resection is the only potential curative treatment (11-13).

Preoperative portal vein embolization (PVE) is nowadays the most accepted treatment before major hepatic resection for HCC in patients with liver fibrosis or cirrhosis and insufficient FLR (14-16). After PVE, the drop-out is approximately $20-30 \%$ of patients due to do lack of adequate hypertrophy of the FLR (as a result of either inadequate regeneration capability in those with cirrhosis or the presence of collaterals) $(17,18)$ or tumor progression during the relatively long waiting time after PVE to achieve adequate FLR volume $(19,20)$. In the last decade, associating liver partition and portal vein ligation for staged hepatectomy (ALPPS) technique has been described to obtain an increase of volume regarding PVE and a decrease of drop out (21). One of the major drawbacks is that it has been associated with a high morbidity and mortality (22) but in recent years the improvement in the selection of patients and the appearance of less invasive modifications from the original technique have improved the results $(23,24)$. However, the use of ALPPS to induce FLR hypertrophy in HCC patients with cirrhosis and chronic liver disease continues to present some uncertainty. We reviewed the literature about ALPPS in HCC in cirrhosis focusing on volumetric date, drop out and postoperative outcomes and then, and we compared this data with the outcomes of PVE in those patients according to the most relevant studies published in this field.

\section{Preoperative assessment of the future liver remnant}

Subsequent early retrospective studies have shown that preoperative assessment of FLR is in fact able to predict hepatic dysfunction in patients undergoing major liver resection (25-27). Considering the liver volume is only a surrogate for liver function and given that liver function may not be homogenous throughout the liver and can be compromised in case of parenchymal liver disease, quantitative functional assessment using regional liver function tests like mebrofenin and albumin scintigraphy has been proposed as a complimentary and possibly a superior methodological approach to prediction of $\operatorname{PHLF}(28,29)$. An alternative to measured FLRV or estimated FLRV is the liver remnant volume to body weight ratio (FRLV-BWR), in which the remnant liver weight is calculated as percentage of body weight. A ratio below $0.5 \%$ (healthy livers) and 0.7 or 1.1 (cirrhotic livers) has been shown to correlate with adverse outcomes (30).

\section{PVE}

From that first series (31), the indications have been significantly extended to make PVE the "gold standard" for patients with large unilobar tumors, with insufficient FLR, and requiring a major hepatectomy. It is a safe procedure and the few alterations produced are probably due to the fact that the hepatocytes in the hepatic "deportalized" lobe experience a process of apoptosis instead of cell necrosis $(32,33)$. On the other hand, the proliferative stimulation represented by the redirection of the portal flow towards the contralateral hepatic lobe induces a growth factormediated hyperplasia. The most potent is the hepatocyte growth factor (HGF) that, together with other mediators, stimulates the production of cytokines such as interleukin 6 and TNF $\alpha$, triggering a gene response that activates the hepatocyte cell cycle and consequently its regeneration (16).

\section{PVE and cirrhosis}

The indication criteria for preoperative PVE for HCC under cirrhosis described by Azoulay et al. in 2000 (34) were: patients younger than 70 years, albumin $\geq 3 \mathrm{~g} / \mathrm{dL}$, total bilirubin $<2 \mathrm{mg} / \mathrm{dL}$, Quick $\geq 80 \%$, Indocyanine Green Retention (ICGR) less than 10 and a FLR lower than $40 \%$. Clinically, the increase of the FLRV in cirrhotic livers after PVE is reported to be in the range of $25 \%$ to $30 \%$, and the hypertrophy ratio of the FLR has also been reported to be approximately 1.3 to $1.5(35,36)$. In a prospective study, Farges et al. (37) suggest that patients with liver cirrhosis prior to partial hepatectomy could benefit from preoperative PVE and recommended performing PVE in patients with right hepatic HCC as a routine preoperative preparation. Recently, Sun et al. (38) compared cirrhotic with non-cirrhotic with PVE and no significant difference was identified between the two groups with respect to FLR volume enlargement at 4-6 weeks following PVE. In a 


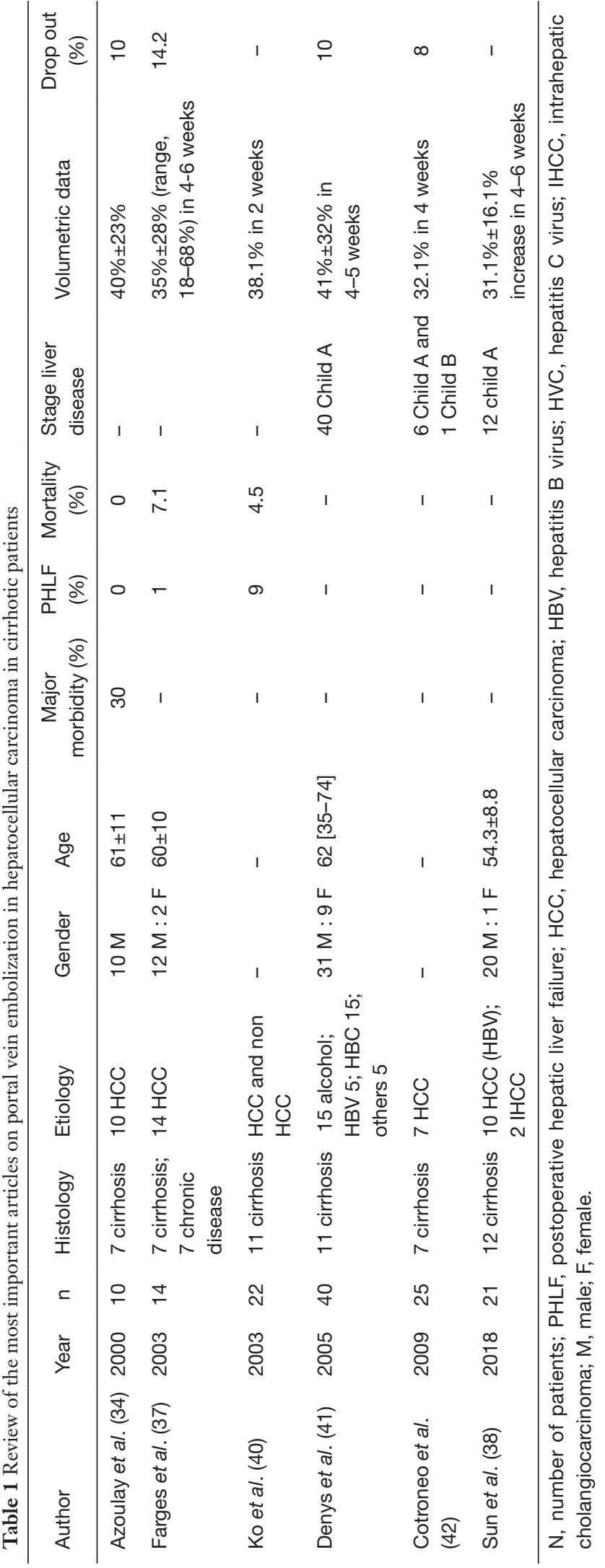

systematic review, the authors (39-42) evaluated the effect of cirrhosis/fibrosis on the hypertrophy response and they described an increase of volume ranging between 24.4$38.4 \%$ in cirrhosis versus $39.4-49.6 \%$ in non-cirrhosis at a median of 36 days. In cirrhotic patients, the drop-out was $20 \%$ with a resectability of $96 \%$ (Table 1).

After PVE liver regeneration may be impaired, causing concerns about tumor progression, this is why some authors speculate to perform a preoperative selective TACE in a standardized sequential manner $(43,44)$. Ogata et al. (45) demonstrated that TACE combined with PVE might effectively induce hypertrophy of FLRs in patients with chronic liver disease and improve the 3 -year disease-free survival rate. In summary, the main advantage of the PVE is achieving a hypertrophy that ranges between $20-50 \%$ in most series, which allows reaching a resectability of $70-100 \%$. However, the two main drawbacks are the risk of tumor progression during the long time of hypertrophy and the absence of liver regeneration. After observing that in most series there are patients with tumor growth, both in the embolized lobe and in the liver.

\section{ALPPS}

The higher and more rapid regeneration of ALPPS than PVE could be related to the occlusion of the intrahepatic circulation, which directs the portal flow to the FLR, associated with venous ischemia of the right lobe. The first publication of the World Registry reported an impressive $80 \%$ increase of volume (49-116\%) in a 7-day interval, with a resectability of $98 \%$, with a total mortality of $9 \%$ and $2 \%$ died after the first stage (46). These results have improved in the last two years due to several factors: refinements in strategic details such as parenchyma splitting (Tourniquet, microwave, partial-ALPPS, mini-ALPPS, laparoscopic), extension of interval to 15 days, testing the in FLR function interval. But, the oncological outcomes remain unclear (24). Recently, dual embolization has been described, performing firstly a right $\mathrm{PVE}$ and after a right hepatic vein embolization but the experience with this technique is still scarce, especially in cirrhotic patients (47).

\section{Short terms outcomes of ALPPS and HCC in cirrbosis}

The use of the ALPPS technique in colorectal liver metastases is the most frequently accepted indication $(48,49)$, being more controversial in primary hepatic tumors, especially in intrahepatic cholangiocarcinoma. 
D'Haese et al. (50) reported higher 90-day mortality among HCC patients than among those with CRLM (31\% vs. $7 \%$ ) and they concluded that the risk associated with ALPPS remains excessive for most HCC patients and that ALPPS should only be performed in a highly selected group of HCC patients younger than 60 years and with a low fibrosis grade. Vennarecci et al. (51) shown ALPPS that it was feasible and safe in HCC patients with cirrhosis and a significant volume increment of FLR can be induced in a short time to allow for completion of the two stage strategy. The same group, in 2016 (52) compared ALPPS in liver cirrhosis (8 HCC) and non-liver cirrhosis (3 CRLM and 1 cholangiocarcinoma). They concluded that ALPPS induced a considerable and comparable FLR growth in HCC patients with liver cirrhosis and patients with CRLM and cholangiocarcinoma with normal liver parenchyma, but the sample size was very small. Chan et al. (53) described in 2016 a safety ALPPS procedure by anterior approach in 17 patients with hepatitis B-related HCC. All patients proceeded to second-stage hepatectomy, after a median of 6 days and a FLR increase of $38.5 \%$. The major morbidity was $11.8 \%$ and hospital mortality rate was $5.9 \%$ and liver histology confirmed chronic hepatitis in 8 patients and cirrhosis in the remaining 9 patients.

Serenari et al. (54) reported a volume increase of $50 \%$ within a median of 7.5 days in 6 cirrhotic livers but one of these 6 patients died within 90 days of ALPPS. In this study, livers from patients who received preoperative chemotherapy (56\%, range: $15-227 \%$ ) or had cirrhosis (50\%, range: $14-178 \%$ ) developed a lower degree of hypertrophy. The authors conclude that, although, there is considerable risk in cirrhotic patients, ALPPS may be a salvage option in selected patients with HCC and fibrosis or cirrhosis and in whom PVE has been unsuccessful. On the other hand, Chan et al. (55) compared complete and partial split in ALPPS and they demonstrate that complete split induced a more significant FLR growth than partial split in chronic hepatitis in terms of daily hypertrophy rate and gain in FLR ratio. The authors also showed that ALPPS could induce FLR hypertrophy in cirrhotic livers within a short period of time. However, even though complete split tended to induce a more rapid FLR hypertrophy than partial split in cirrhosis (hypertrophy rate 32.2 vs. $16.9 \mathrm{~mL} /$ day) the difference was less obvious for cirrhotic livers (FLR\% increment: $14.8 \%$ vs. $11.0 \%$ ) than for chronic hepatitis (FLR\% increment: $18.1 \%$ vs. $11.3 \%$ ).

In terms of ALPPS for HBV-related HCC the results are controversial. Cai et al. (56) described worst outcomes for
HBV-related HCC with cirrhosis. They showed that FLRs of cirrhotic liver do hypertrophy with an increase of $28.1 \%$, but it took longer for the FLR to reach to a safe volume to undergo the second operation. Half of the patients died (two of them after the first stage) because of postoperative liver failure or other complications, which emphasizes the need of optimal patient selection to reduce the mortality. The subgroup analysis merely demonstrated that too small FLR $(<30 \%)$ before operation contributed to a tragic outcome, while the FLR/SLV between $30 \%$ and $40 \%$ presented a satisfactory result. On the other hand, Wang et al. (33) analyzed the ALPPS outcomes in 45 patients with unresectable hepatitis B virus-related HCC with better results. The majority of patients presented a BCLC A $(42.2 \%)$ and the grades of liver fibrosis were absence (4.4\%), low (22.2\%), moderate $(24.4 \%)$, severe $(11.1 \%)$ and cirrhosis (28.9\%). They concluded that ALPPS could induce enough volume of the FLR to increase to allow liver resection in HCC patients and that the rate of hypertrophy of the FLR negatively correlated with the severity of fibrosis/cirrhosis. In this study, the hypertrophy of FLR in the normal liver was attributed to both regeneration and increased size of hepatocytes, whereas hypertrophy of FLR in the advanced fibrosis liver mainly relies on increase of the size of the hepatocyte (Table 2).

Due to the scarce literature on this topic, in series of published clinical cases that have described the results of ALPPS in HCC, we have analyzed those that describe the histopathological findings (58-64). A total of 7 cases were registered ( 5 of cirrhosis and 2 of moderate fibrosis). There was no mortality and only one patient presented small for size, probably because the FLR prior to the second time was $24 \%$. With the exception of this case, the rest of the patients reached an FLR between $29 \%$ and $61 \%$ in a time range between 4 and 20 days (Table 3).

\section{Long terms outcomes of ALPPS and HCC in cirrbosis}

Few authors detail the follow-up results and the patients included in the follow-up are scarce. Senerari et al. (54) describe an overall survival (OS) of $75 \%$ and a disease free survival of $62 \%$ at 1 year. Wang et al. (33) published an OS rate at 1 - and 3 -year of $64.2 \%$ and $61.2 \%$ whereas the DFS rates at 1 - and 3 -year were $64.2 \%$ and $61.2 \%$, respectively. On the study by Cai et al. (56) at a mean follow-up of 16 months, 1 patient died of upper gastrointestinal hemorrhage at 4 month and another patient died of recurrence and lung metastasis at the 13 months, and 4 


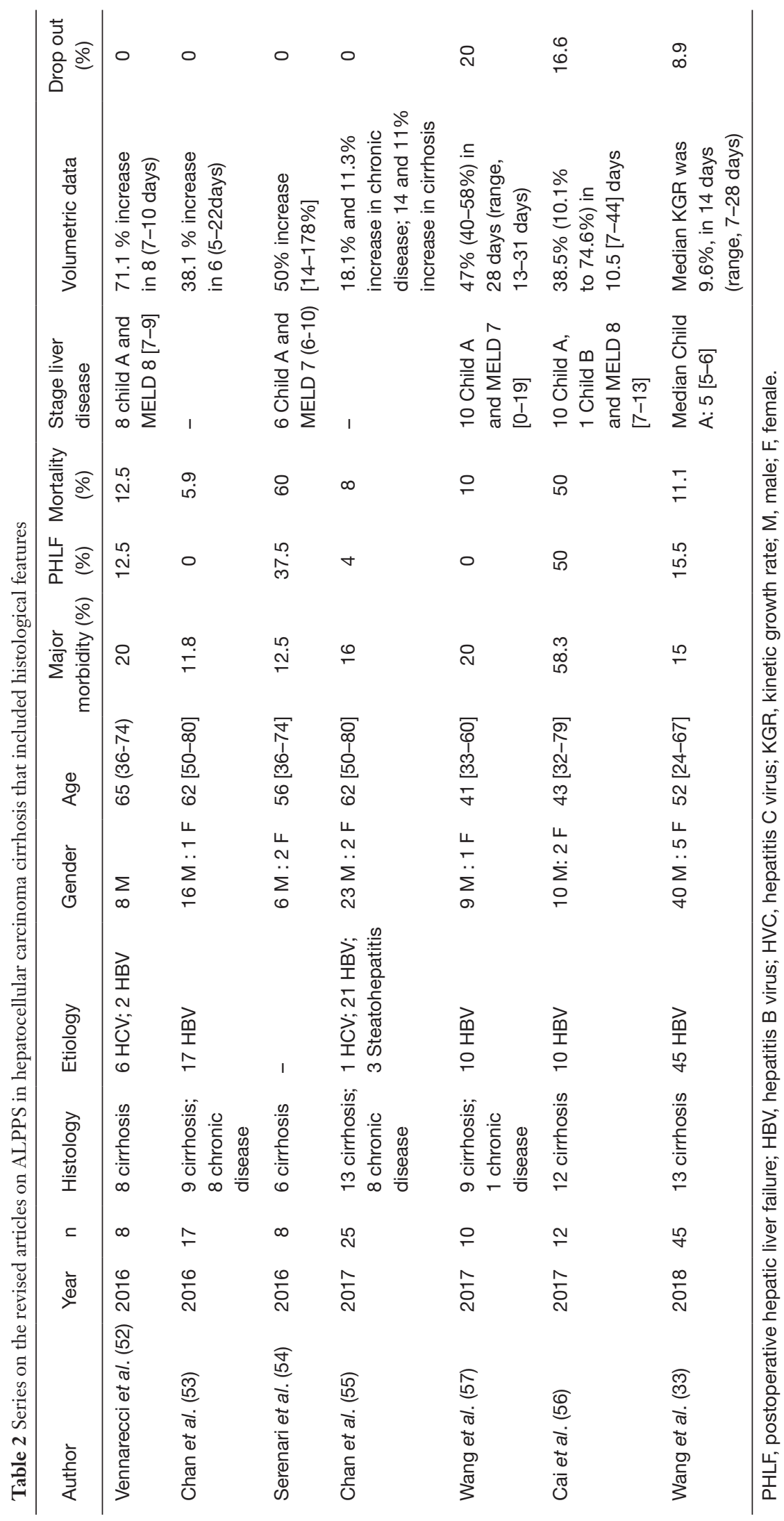


Table 3 Clinical cases on the revised articles on ALPPS in hepatocellular cirrhosis that included histological features

\begin{tabular}{|c|c|c|c|c|c|c|c|c|c|}
\hline Author & Year & Histology & Etiology & Gender & Age & Morbidity & PHLF & Mortality & FLR \\
\hline Xiao et al. (59) & 2015 & Cirrhosis & - & - & - & No & No & No & $27 \%$ to $40.6 \%$ in 13 days \\
\hline de Santibañes et al. (61) & 2016 & Cirrhosis & - & $\mathrm{F}$ & 66 & No & No & No & $40 \%$ to $61 \%$ in 10 days \\
\hline Papamichail et al. (62) & 2016 & Cirrhosis & Alcohol & M & 68 & No & $\begin{array}{l}\text { Small for } \\
\text { size }\end{array}$ & No & $14 \%$ to $24 \%$ in 10 days \\
\hline Chen et al. (63) & 2016 & Cirrhosis & HBV & M & 43 & No & No & No & $29.1 \%$ to $51.2 \%$ in 20 days \\
\hline
\end{tabular}

PHLF, postoperative hepatic liver failure; HBV, hepatitis B virus; HVC, hepatitis C virus; FLR, future liver remnant; $M$, male; F, female.

out 6 were still alive and 2 were live and free of disease. In the series $(\mathrm{n}=10)$ of Wang et al. (57), the 2 patients who did not complete de stage 2 died in the following 3 months after discharge. Another two cases had a tumor recurrence within 3 months of discharge and 1 of these died and another underwent TACE with Sorafenib. Another patient subsequently underwent TACE when local tumor recurrence was observed at 9 months and 4 cases had no disease recurrence.

\section{Role of ALPPS variants modifications in HCC}

The variants of the original ALPPS technique have allowed to improve the results and this improvement in the morbidity it could be useful in high risk patients such as cirrhotic patients. All the variants share the same theoretical concept from the original technique. The only novel aspect that these variants introduce concerns the anatomical place of the bipartition and, therefore, the type of hepatectomy that will be carried out during the second surgical time. These alternatives are based on two truly novel and different concepts with respect to classical ALPPS and consist of not splitting the hepatic parenchyma (ALPPS-Tourniquet, radiofrequency ablation, microwave ablation) or splitting the parenchyma partially (partial ALPPS, Mini ALPPS). The first variant described was our technique [2011] named as Tourniquet-ALPPS or ALTPS. We have operated on 4 HCC treated with Tourniquet-ALPPS (2 with cirrhosis and 2 with low fibrosis). Despite being a very small sample, both groups presented a similar FLR $(42.5 \%$ and $105 \%$ vs. $51.7 \%$ and $187.5 \%$, respectively) in the same period without mortality, with a major complication in each group and without drop out. Wang et al. (57) described RALPPS technique in cirrhosis-related HCC from HBV. Nine of 10 patients included in the study present with cirrhosis. Two patients did not proceed to the second-stage operation: one patient had liver dysfunction and massive ascites after stage I, and the other patient had metastasis in FLR tissue during the waiting period before stage II. The median FLR before stage I was $31 \%$ (364 mL) with an increased to $47 \%$ $(632 \mathrm{~mL})$ before stage II after a median interval of 28 days and a median percentage increase in FLR of $53 \%(210 \mathrm{~mL})$. The morbidity and mortality results were poor with a PHLF in 5 of 10 patients and mortality in 5 of 10 patients. The results were related due to the patients were operated on in the II stage with and insufficient FLR volume for a liver cirrhosis.

\section{ALPPS and bistology features}

Regarding liver histology before the first intervention, it is considered that patients with fibrosis have worse regeneration and that it is specially related to the degree of fibrosis. There are discrepancies regarding the regeneration capacity of the liver when it presents with fibrosis, cirrhosis, cholestasis, macrosteatosis and alterations related to chemotherapy (Figure 1). Furthermore, there are no data regarding the influence of histological alterations before the second intervention and the presence of postoperative hepatic insufficiency. Several studies have been able to establish a histological correlation with the increase of FLR in patients with ALPPS by using 

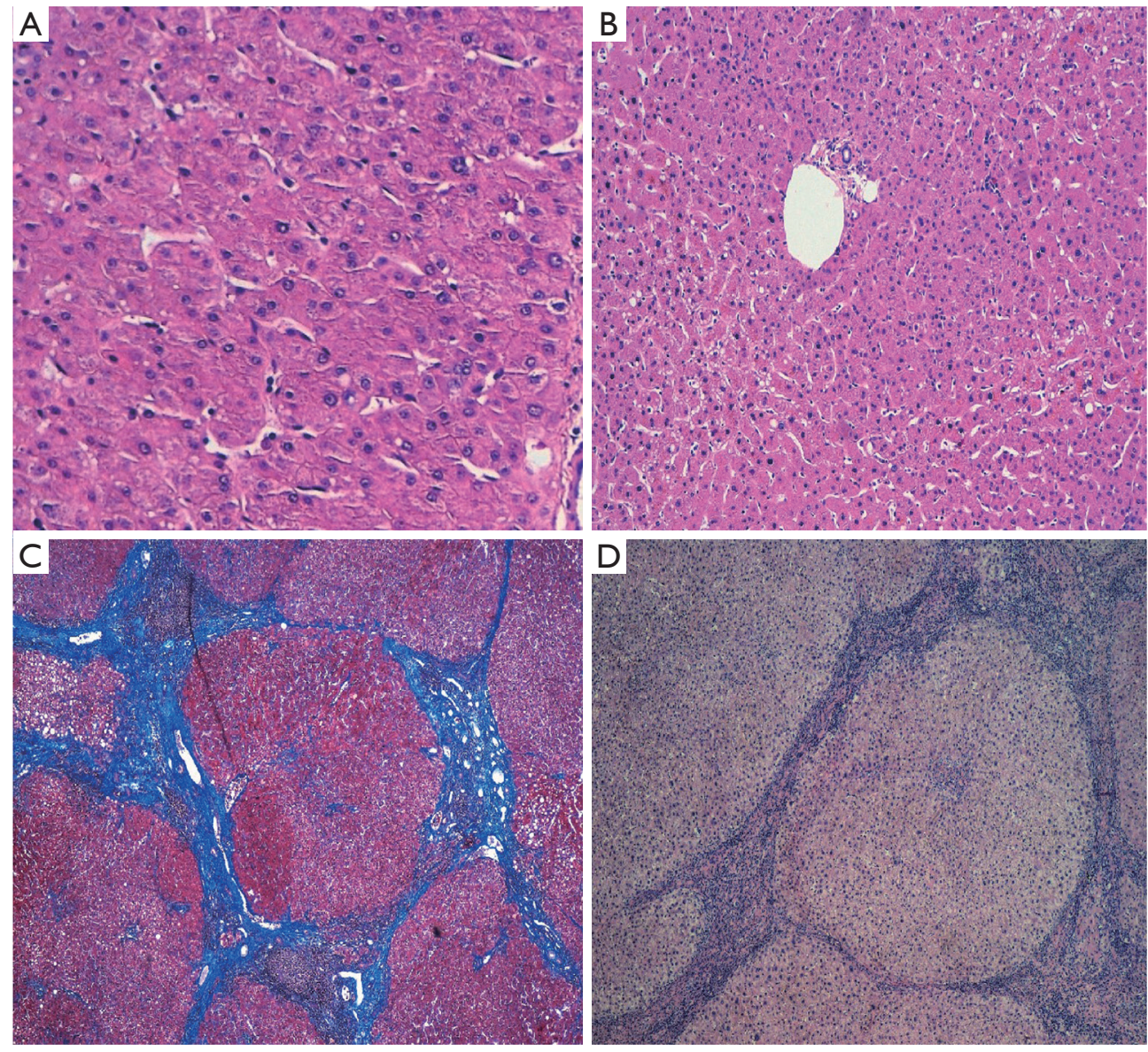

Figure 1 Biopsies in the future liver remnant in a liver without alterations, before (A) and after (B) Tourniquet ALPPS (H\&E, 100x). The normal architecture of the liver is preserved in both and there are no significant differences between them. (C) Second stage biopsy of cirrhotic liver (Masson's Trichrome, 40x) after Tourniquet ALPPS. In contrast to the previous images, the normal architecture of the liver is replaced by multiple regeneration nodes separated from each other by fibrous septa, and no relevant differences can be highlighted when compared with a cirrhotic liver previous to the surgery (D, H\&E 40x).

immunohistochemical techniques such as $\mathrm{Ki}-67$ for the measurement of hepatocyte cell proliferation index (65-69). The underlying biological substrate is not well understood, but it is suggested that a combination of factors including hemodynamic changes or damage caused by the surgery itself would be able to induce a systemic response mediated by growth factors, transcription and cytokines that would ultimately lead to the activation and proliferation of hepatocytes $(66,70)$. Histologically, differences have been observed with respect to PVE $(69,70)$, as an increase in the hepatocyte density or a decrease in the size of the hepatocyte, as well as a greater cellular atrophy, degeneration, necrosis, fibrosis or sinusoidal dilatation in the deportalized areas, as well as congestion. The latter would be of special relevance to explain the higher proliferation rates reported in ALPPS. However, little has been studied about ALPPS in a cirrhotic liver. Cirrhosis is the last phase of a dynamic diffuse fibrosing process in which the normal architecture of the liver is replaced by a nodular pattern as a result of liver damage, usually chronic (71). The fibrotic tissue ends up hindering liver regeneration (72). Although previous cirrhosis would limit in principle the process of hypertrophy, the degree of hypertrophy induced by ALPPS could be beneficial even for cirrhotic patients $(59,73,74)$. However, discordance between the growth in liver volume in ALPPS and the functional growth of the proliferated liver has been detected, with multiple signs of immaturity in the tissue being appreciated $(73,75-77)$. Whether these discrepancies are transient or not, this means that care must be taken in patients with a low previous liver remnant, 
as well as in cirrhotic patients. It is unknown if this discordance persists also in modified ALPPS techniques, since there are practically no studies that address liver function for this technique (76). To solve these unknowns, different animal models are being developed for the study and understanding of this set of processes (78-81), included a rat model with fibrosis.

\section{Future directions}

Major liver resections in cirrhotic livers continue to be complex interventions due to the high risk of PHLF and mortality. The most important and key aspect to obtain good results in this subpopulation of patients is to achieve a hepatic remnant with enough size to perform the surgery with the greatest possible safety. The study of the patient should be as complete as possible, therefore it is recommended that along with the volumetric and the ICGR15 rate, the RLV-BWR and the quantitative functional assessment using regional liver function tests like mebrofenin and albumin scintigraphy should also be performed. The patients in which all these measurements are favorable, surgery could be performed with the lowest probability of failure. Regarding the type of approach in these patients with a cirrhotic liver, the PVE has traditionally been associated with or not associated with TACE as a step prior to surgery, but the appearance of the ALPPS technique offers a new alternative. The great advantage of this technique is that it provides us with high rates of resectability due to its large volumetric capacity in a short time compared to the PVE. The initial problems of this technique on the morbidity and mortality results have decreased drastically due to a better selection of patients, learning curve and less aggressive techniques variations in the first time that provide the same results of volumetric increase. For this reason, ALPPS is a feasible technique in patients selected with HCC and cirrhosis, and it could be performed in an experienced center as a technique of first option in cases with a very low FLR or as a rescue technique in the face of PVE failure.

\section{Acknowledgments}

None.

\section{Footnote}

Conflicts of Interest: The authors have no conflicts of interest to declare.
Ethical Statement: The authors are accountable for all aspects of the work in ensuring that questions related to the accuracy or integrity of any part of the work are appropriately investigated and resolved.

\section{References}

1. Wong MC, Jiang JY, Goggins WB, et al. International incidence and mortality trends of liver cancer: a global profile. Sci Rep 2017;7:45846.

2. Llovet JM, Zucman-Rossi J, Pikarsky E, et al. Hepatocellular carcinoma. Nature Reviews Disease Primers 2016;2:16018.

3. Ghouri YA, Mian I, Rowe JH. Review of hepatocellular carcinoma: Epidemiology, etiology, and carcinogenesis. J Carcinog 2017;16:1.

4. Balogh J, Victor D 3rd, Asham EH, et al. Hepatocellular carcinoma: a review. J Hepatocell Carcinoma 2016;3:41-53.

5. Heimbach JK, Kulik LM, Finn RS, et al. AASLD guidelines for the treatment of hepatocellular carcinoma. Hepatology 2018;67:358-80.

6. Vogel A, Cervantes A, Chau I, et al. Hepatocellular carcinoma: ESMO Clinical Practice Guidelines for diagnosis, treatment and follow-up. Ann Oncol 2018;29:iv238-iv255.

7. Yu SJ. A concise review of updated guidelines regarding the management of hepatocellular carcinoma around the world: 2010-2016. Clin Mol Hepatol 2016;22:7-17.

8. Arroyo V, Moreau R, Kamath PS, et al. Acute-onchronic liver failure in cirrhosis. Nat Rev Dis Primers 2016;2:16041.

9. Guglielmi A, Ruzzenente A, Conci S, et al. How much remnant is enough in liver resection? Dig Surg 2012;29:6-17.

10. Han K, Kim JH. Transarterial chemoembolization in hepatocellular carcinoma treatment: Barcelona clinic liver cancer staging system. World J Gastroenterol 2015;21:10327-35.

11. Hackl C, Schlitt HJ, Renner P, et al. Liver surgery in cirrhosis and portal hypertension. World J Gastroenterol 2016;22:2725-35.

12. Ho MC, Hasegawa K, Chen XP, et al. Surgery for Intermediate and Advanced Hepatocellular Carcinoma: A Consensus Report from the 5th Asia-Pacific Primary Liver Cancer Expert Meeting (APPLE 2014). Liver Cancer 2016;5:245-56.

13. Kapitanov T, Neumann UP, Schmeding M. Hepatocellular 
Carcinoma in Liver Cirrhosis: Surgical Resection versus Transarterial Chemoembolization-A Meta-Analysis. Gastroenterol Res Pract 2015;2015:696120.

14. Yifan T, Ming X, Yifan W, et al. Hepatic regeneration by associating liver partition and portal vein ligation for staged hepatectomy (ALPPS) is feasible but attenuated in rat liver with thioacetamide-induced fibrosis. Surgery 2019;165:345-52.

15. Aoki T, Kubota K. Preoperative portal vein embolization for hepatocellular carcinoma: Consensus and controversy. World J Hepatol 2016;8:439-45.

16. Madoff DC, Abdalla EK, Vauthey JN. Portal vein embolization in preparation for major hepatic resection: evolution of a new standard of care. J Vasc Interv Radiol 2005;16:779-90.

17. Brouquet A, Abdalla EK, Kopetz S, et al. High survival rate after two-stage resection of advanced colorectal liver metastases: response-based selection and complete resection define outcome. J Clin Oncol 2011;29:1083-90.

18. Narita M, Oussoultzoglou E, Jaeck D, et al. Twostage hepatectomy for multiple bilobar colorectal liver metastases. Br J Surg 2011;98:1463-75.

19. Kokudo N, Tada K, Seki M, et al. Proliferative activity of intrahepatic colorectal metastases after preoperative hemihepatic portal vein embolization. Hepatology 2001;34:267-72

20. Simoneau E, Aljiffry M, Salman A, et al. Portal vein embolization stimulates tumour growth in patients with colorectal cancer liver metastases. HPB (Oxford) 2012;14:461-8.

21. Lang H, de Santibanes E, Schlitt HJ, et al. 10th Anniversary of ALPPS-Lessons Learned and quo Vadis. Ann Surg 2019;269:114-9.

22. Schnitzbauer AA, Lang SA, Goessmann H, et al. Right portal vein ligation combined with in situ splitting induces rapid left lateral liver lobe hypertrophy enabling 2-staged extended right hepatic resection in small-for-size settings. Ann Surg 2012;25 5:405-14.

23. Linecker M, Stavrou GA, Oldhafer KJ, et al. The ALPPS Risk Score: Avoiding Futile Use of ALPPS. Ann Surg 2016;264:763-71.

24. Linecker M, Bjornsson B, Stavrou GA, et al. Risk Adjustment in ALPPS Is Associated With a Dramatic Decrease in Early Mortality and Morbidity. Ann Surg 2017;266:779-86.

25. Thirunavukarasu P, Aloia TA. Preoperative Assessment and Optimization of the Future Liver Remnant. Surg Clin North Am 2016;96:197-205.
26. Vauthey JN, Chaoui A, Do KA, et al. Standardized measurement of the future liver remnant prior to extended liver resection: methodology and clinical associations. Surgery 2000;127:512-9.

27. Pulitano C, Crawford M, Joseph D, et al. Preoperative assessment of postoperative liver function: the importance of residual liver volume. J Surg Oncol 2014;110:445-50.

28. Chapelle T, Op De Beeck B, Huyghe I, et al. Future remnant liver function estimated by combining liver volumetry on magnetic resonance imaging with total liver function on (99m)Tc-mebrofenin hepatobiliary scintigraphy: can this tool predict post-hepatectomy liver failure? HPB (Oxford) 2016;18:494-503.

29. Mizutani Y, Hirai T, Nagamachi S, et al. Prediction of Posthepatectomy Liver Failure Proposed by the International Study Group of Liver Surgery: Residual Liver Function Estimation With 99m Tc-Galactosyl Human Serum Albumin Scintigraphy. Clin Nucl Med 2018;43:77-81.

30. Goumard C, Perdigao F, Cazejust J, et al. Is computed tomography volumetric assessment of the liver reliable in patients with cirrhosis? HPB (Oxford) 2014;16:188-94.

31. Nagino M, Kamiya J, Kanai M, et al. Right trisegment portal vein embolization for biliary tract carcinoma: technique and clinical utility. Surgery 2000;127:155-60.

32. Duncan JR, Hicks ME, Cai SR, et al. Embolization of portal vein branches induces hepatocyte replication in swine: a potential step in hepatic gene therapy. Radiology 1999;210:467-77.

33. Wang Z, Peng Y, Hu J, et al. Associating Liver Partition and Portal Vein Ligation for Staged Hepatectomy for Unresectable Hepatitis B Virus-related Hepatocellular Carcinoma: A Single Center Study of 45 Patients. Ann Surg 2018. [Epub ahead of print].

34. Azoulay D, Castaing D, Krissat J, et al. Percutaneous portal vein embolization increases the feasibility and safety of major liver resection for hepatocellular carcinoma in injured liver. Ann Surg 2000;232:665-72.

35. Soggiu F, Giovinazzo F, Straiton J, et al. Monosegment ALPPS hepatectomy preserving segment 4 for colorectal liver metastases: literature review and our experience. Hepatobiliary Surg Nutr 2018;7:105-15.

36. Krishnamurthy J, Naragund AV, Mahadevappa B. First Ever Robotic Stage One ALPPS Procedure in India: for Colorectal Liver Metastases. Indian J Surg 2018;80:269-71.

37. Farges O, Belghiti J, Kianmanesh R, et al. Portal vein embolization before right hepatectomy: prospective 
clinical trial. Ann Surg 2003;237:208-17.

38. Sun JH, Zhang YL, Nie CH, et al. Effects of liver cirrhosis on portal vein embolization prior to right hepatectomy in patients with primary liver cancer. Oncol Lett 2018;15:1411-6.

39. van Lienden KP, van den Esschert JW, de Graaf W, et al. Portal vein embolization before liver resection: a systematic review. Cardiovasc Intervent Radiol 2013;36:25-34.

40. Ko GY, Sung KB, Yoon HK, et al. Preoperative portal vein embolization with a new liquid embolic agent. Radiology 2003;227:407-13.

41. Denys A, Lacombe C, Schneider F, et al. Portal vein embolization with N-butyl cyanoacrylate before partial hepatectomy in patients with hepatocellular carcinoma and underlying cirrhosis or advanced fibrosis. J Vasc Interv Radiol 2005;16:1667-74.

42. Cotroneo AR, Innocenti P, Marano G, et al. Prehepatectomy portal vein embolization: single center experience. Eur J Surg Oncol 2009;35:71-8.

43. Ronot M, Cauchy F, Gregoli B, et al. Sequential transarterial chemoembolization and portal vein embolization before resection is a valid oncological strategy for unilobar hepatocellular carcinoma regardless of the tumor burden. HPB (Oxford) 2016;18:684-90.

44. Yoo H, Kim JH, Ko GY, et al. Sequential transcatheter arterial chemoembolization and portal vein embolization versus portal vein embolization only before major hepatectomy for patients with hepatocellular carcinoma. Ann Surg Oncol 2011;18:1251-7.

45. Ogata S, Belghiti J, Farges O, et al. Sequential arterial and portal vein embolizations before right hepatectomy in patients with cirrhosis and hepatocellular carcinoma. Br J Surg 2006;93:1091-8.

46. Schadde E, Raptis DA, Schnitzbauer AA, et al. Prediction of Mortality After ALPPS Stage-1: An Analysis of 320 Patients From the International ALPPS Registry. Ann Surg 2015;262:780-5; discussion 785-6.

47. Le Roy B, Perrey A, Fontarensky M, et al. Combined Preoperative Portal and Hepatic Vein Embolization (Biembolization) to Improve Liver Regeneration Before Major Liver Resection: A Preliminary Report. World J Surg 2017;41:1848-56.

48. Hernandez-Alejandro R, Bertens KA, Pineda-Solis $\mathrm{K}$, et al. Can we improve the morbidity and mortality associated with the associating liver partition with portal vein ligation for staged hepatectomy (ALPPS) procedure in the management of colorectal liver metastases? Surgery
2015;157:194-201.

49. Lang H, Baumgart J, Mittler J. Associating Liver Partition and Portal Vein Ligation for Staged Hepatectomy in the Treatment of Colorectal Liver Metastases: Current Scenario. Dig Surg 2018;35:294-302.

50. D'Haese JG, Neumann J, Weniger M, et al. Should ALPPS be Used for Liver Resection in Intermediate-Stage HCC? Ann Surg Oncol 2016;23:1335-43.

51. Vennarecci G, Laurenzi A, Santoro R, et al. The ALPPS procedure: a surgical option for hepatocellular carcinoma with major vascular invasion. World J Surg 2014;38:1498-503.

52. Vennarecci G, Grazi GL, Sperduti I, et al. ALPPS for primary and secondary liver tumors. Int J Surg 2016;30:38-44.

53. Chan AC, Poon RT, Chan C, et al. Safety of ALPPS Procedure by the Anterior Approach for Hepatocellular Carcinoma. Ann Surg 2016;263:e14-6.

54. Serenari M, Zanello M, Schadde E, et al. Importance of primary indication and liver function between stages: results of a multicenter Italian audit of ALPPS 2012-2014. HPB (Oxford) 2016;18:419-27.

55. Chan AC, Chok K, Dai JW, et al. Impact of split completeness on future liver remnant hypertrophy in associating liver partition and portal vein ligation for staged hepatectomy (ALPPS) in hepatocellular carcinoma: Complete-ALPPS versus partial-ALPPS. Surgery 2017;161:357-64.

56. Cai X, Tong Y, Yu H, et al. The ALPPS in the Treatment of Hepatitis B-Related Hepatocellular Carcinoma With Cirrhosis: A Single-Center Study and Literature Review. Surg Innov 2017;24:358-64.

57. Wang Q, Yan J, Feng X, et al. Safety and efficacy of radiofrequency-assisted ALPPS (RALPPS) in patients with cirrhosis-related hepatocellular carcinoma. Int J Hyperthermia 2017;33:846-52.

58. Chia NH, Lai EC, Lau WY. Associating liver partition and portal vein ligation for a patient with hepatocellular carcinoma with a background of hepatitis B related fibrotic liver. Int J Surg Case Rep 2014;5:1077-81.

59. Xiao L, Li JW, Zheng SG. Totally laparoscopic ALPPS in the treatment of cirrhotic hepatocellular carcinoma. Surg Endosc 2015;29:2800-1.

60. Cheung TT, Wong TCL, Chan SC. Technical note en ALPPS fer a patient with advanced hepatocellular carcinoma associated with invasion of the inferior vena cava. Hepatobiliary \& Pancreatic Diseases International 2016;15:319-23. 
61. de Santibañes E, Alvarez FA, Ardiles V, et al. Inverting the ALPPS paradigm by minimizing first stage impact: the Mini-ALPPS technique. Langenbecks Arch Surg 2016;401:557-63.

62. Papamichail M, Pizanias M, Yip V, et al. Associating liver partition and portal vein ligation for staged hepatectomy (ALPPS) procedure for hepatocellular carcinoma with chronic liver disease: a case report and review of literature. Korean J Hepatobiliary Pancreat Surg 2016;20:75-80.

63. Chen JX, Ran HQ, Sun CQ. Associating microwave ablation and portal vein ligation for staged hepatectomy for the treatment of huge hepatocellular carcinoma with cirrhosis. Ann Surg Treat Res 2016;90:287-91.

64. Cavaness KM, Doyle MB, Lin Y, et al. Using ALPPS to induce rapid liver hypertrophy in a patient with hepatic fibrosis and portal vein thrombosis. J Gastrointest Surg 2013;17:207-12.

65. Schlegel A, Lesurtel M, Melloul E, et al. ALPPS: from human to mice highlighting accelerated and novel mechanisms of liver regeneration. Ann Surg 2014;260:83946; discussion 846-7.

66. García-Pérez R, Revilla-Nuin B, Martinez CM, et al. Associated Liver Partition and Portal Vein Ligation (ALPPS) vs Selective Portal Vein Ligation (PVL) for Staged Hepatectomy in a Rat Model. Similar Regenerative Response? PLoS One 2015;10:e0144096.

67. Dhar DK, Mohammad GH, Vyas S, et al. A novel rat model of liver regeneration: possible role of cytokine induced neutrophil chemoattractant- 1 in augmented liver regeneration. Ann Surg Innov Res 2015;9:11.

68. Wei W, Zhang T, Zafarnia S, et al. Establishment of a rat model: Associating liver partition with portal vein ligation for staged hepatectomy. Surgery 2016;159:1299-307.

69. Matsuo K, Murakami T, Kawaguchi D, et al. Histologic features after surgery associating liver partition and portal vein ligation for staged hepatectomy versus those after hepatectomy with portal vein embolization. Surgery 2016;159:1289-98.

70. Moris D, Vernadakis S, Papalampros A, et al. Mechanistic insights of rapid liver regeneration after associating liver partition and portal vein ligation for stage hepatectomy. World J Gastroenterol 2016;22:7613-24.

71. Tampi C. Pathology for the HPB Surgeon. Indian J Surg 2012;74:67-72.

72. Cordero-Espinoza L, Huch M. The balancing act of the liver: tissue regeneration versus fibrosis. J Clin Invest 2018;128:85-96.

73. Tong YF, Meng N, Chen MQ, et al. Maturity of associating liver partition and portal vein ligation for staged hepatectomy-derived liver regeneration in a rat model. World J Gastroenterol 2018;24:1107-19.

74. Cai X, Peng S, Duan L, et al. Completely laparoscopic ALPPS using round-the-liver ligation to replace parenchymal transection for a patient with multiple right liver cancers complicated with liver cirrhosis. J Laparoendosc Adv Surg Tech A 2014;24:883-6.

75. Tanaka K, Matsuo K, Murakami T, et al. Associating liver partition and portal vein ligation for staged hepatectomy (ALPPS): short-term outcome, functional changes in the future liver remnant, and tumor growth activity. Eur J Surg Oncol 2015;41:506-12.

76. Kang D, Schadde E. Hypertrophy and Liver Function in ALPPS: Correlation with Morbidity and Mortality. Visc Med 2017;33:426-33.

77. Matsuo K, Hiroshima Y, Yamazaki K, et al. Immaturity of Bile Canalicular-Ductule Networks in the Future Liver Remnant While Associating Liver Partition and Portal Vein Occlusion for Staged Hepatectomy (ALPPS). Ann Surg Oncol 2017;24:2456-64.

78. Shi JH, Hammarstrom C, Grzyb K, et al. Experimental evaluation of liver regeneration patterns and liver function following ALPPS. BJS Open 2017;1:84-96.

79. Shi H, Yang G, Zheng T, et al. A preliminary study of ALPPS procedure in a rat model. Sci Rep 2015;5:17567.

80. Croome KP, Mao SA, Glorioso JM, et al. Characterization of a porcine model for associating liver partition and portal vein ligation for a staged hepatectomy. HPB (Oxford) 2015;17:1130-6.

81. Schadde E, Hertl M, Breitenstein S, et al. Rat Model of the Associating Liver Partition and Portal Vein Ligation for Staged Hepatectomy (ALPPS) Procedure. J Vis Exp 2017. doi: $10.3791 / 55895$.

Cite this article as: Lopez-Lopez V, Robles-Campos R, Brusadin R, Lopez-Conesa A, de la Peña J, Caballero A, Arevalo-Perez J, Navarro-Barrios A, Gómez P, Parrilla-Paricio P. ALPPS for hepatocarcinoma under cirrhosis: a feasible alternative to portal vein embolization. Ann Transl Med 2019;7(22):691. doi: 10.21037/atm.2019.10.57 